\title{
"Did you tell your mum?"
}

\author{
Isabelle Larrivée
}

Children's literature reveals a common reaction to death: we wish to hide it, to hush it, we attempt to forget its existence - especially around children for whom, we believe, the reality is too difficult to grasp. However, what is hidden tends to reveal itself with increased force, and the child's reality brings back the dead everyone so desperately tried to burry. Is it possible for adults to imagine that nothing escapes a child's awareness, especially not a secret? Even the dead have a right to exist.

La question de la mort parsème la littérature. Elle est à l'œuvre partout, si elle n'est pas l'œuvre elle-même et sans elle, il n'y aurait sans doute pas de littérature.

Pas de littérature sans la question de la mort qui y travaille? On peut se demander alors de quoi nous entretient généralement la littérature pour la jeunesse, ou plutôt de quoi elle n'entretient pas les enfants. Elle nous parle de petits garçons bien tannants qui jouent de vilains tours à une grand-mère éternelle, de petites filles qui se transforment en détectives de thrillers sans cadavre, bref, rares sont les textes où, lorsqu'ils s'y prêteraient, la question de la mort est abordée comme étant une question contiguë à la vie. C'est un peu comme on le fait dans les familles, en somme, parce que l'on s'imagine qu'il serait sordide de parler de la mort à un enfant, parce que la mort n'est pas un sujet que l'on doit aborder avec lui. Et puisque ce n'est pas un sujet que l'on aborde avec lui, c'est généralement lui qui nous interpelle sur cette question. Encore faut-il savoir entendre, et savoir répondre.

Ce qui est sordide, c'est de ne pas vouloir en parler. C'est de taire la mort. C'est de faire comme si elle n'existait pas. Il y a une évidente pudeur à évoquer la question de la mort, ou à rappeler le souvenir d'un disparu. La vie devient ainsi un endless story, dans laquelle la conscience qu'ont tous les enfants de la mort s'abîme, abruptement contredite, et finit par prendre l'allure d'un mensonge. Or, le vrai mensonge est celui qu'un silence contrit impose à une histoire familiale, la privant de sa texture, de la richesse qu'elle recèle, et des outils qu'elle offre pour se positionner face à l'existence.

En ce sens, que peut dire une enfant face à une mère physiquement morte, surtout si elle n'en a aucun souvenir, si elle ne l'a pas connue? Que peut l'enfant face au silence des adultes, cet évitement quotidien? Forcément, elle sait qu'il y a eu mort. Elle sait qu'une mère jadis exista qui la porta en elle. Mais cette mère gommée par la réserve et la retenue des adultes, refait sa route vers la vie, et doit tôt ou tard rejaillir, par des détours parfois inusités. C'est désormais l'enfant qui porte sa mère et qui devra la porter au jour. Il n'y a pas de silence possible quant 
à la mort. Il ne saurait y avoir de secret. Donner aux morts le droit d'exister, leur rendre une vie symbolique à laquelle ils ont droit dans la mémoire tronquée des enfants, voilà de quoi participe le propos de Jacqueline Wilson dans son récit The Cat Mummy (2001).

Verity, c'est le nom de la jeune narratrice, et qui détermine bien évidemment le personnage, n'a pas connu sa mère, morte le jour de sa naissance. Elle vit avec son père et ses grands-parents maternels. On n'évoque pas le souvenir de la mère dans cette famille. Tout le monde a trop souffert de sa mort, et mieux vaut après tout ne pas en parler, ne plus en parler.

Verity vit aussi avec Mabel, la chatte ayant appartenu à sa mère, qui servira dans le récit de substitut maternel à divers niveaux.

Mabel est d'abord présentée ainsi par Verity:

I have a pet. She is a Tabby cat called Mabel. I love her dearly. But she is very, very, very boring. She doesn't do anything. She just sleeps. Sometimes, I leave her curled on my bed when I go to school and when I come home there she still is, in exactly the same position... (9)

Cette chatte est donc d'emblée décrite comme une sorte de « Mabel au bois dormant ", dans toute l'immobilité et la fixité de la mort. Elle ne bouge pas, et cela ennuie profondément Verity. Elle serait ainsi le prolongement d'une mère morte incorporée, c'est-à-dire devenue objet d'amour fantasmatique, le fantasme se greffant ici sur le vacuum absolu de la mémoire familiale vis-à-vis de la mère. Morte, en somme, apparemment morte, mais toujours bien vivante, ou ayant laissé derrière elle, tel un fossile, la trace du vivant. Mabel se tient donc dans la continuité affective de la mère, ayant été aimée par elle et l'étant aujourd'hui par sa fille. Elle est la cheville ouvrière garantissant à la fois la symbolisation de la mère et la possibilité du deuil.

Mabel est ainsi la dépositaire du secret familial:

I hate thinking about my mum being buried.

I try to imagine her alive instead. I'll tell you a very private secret. I sometimes talk about my mum to Mabel, because Mabel doesn't ever get upset.

I talk and talk and talk about my mum. Mabel listens. When she's not asleep. (13)

Un jour toutefois, il fallait bien s'y attendre et la grand-mère avait de longue date préparé Verity à cette fatalité inéluctable, Mabel disparaît et les adultes de la maison soupçonnent sa mort. Cette disparition provoque chez Verity un grand chagrin et une grande inquiétude. Là se vit pour elle la douleur de la séparation et le début d'un deuil véritable: « I wished I could hold her in my arms and tell her just how much she meant to me $»(25)$. Des recherches ont lieu, dans le cadre limité de la maison, d'abord, puis dans le jardin, enfin dans le quartier. Préoccupée par toutes 
ces recherches, Verity est poursuivie la nuit par des cauchemars, et assiste distraite à la classe.

Tant que le chat était là, il servait de rempart sacré contre le deuil, tout comme Verity l'étudie à l'école, dans ses cours sur l'Égypte ancienne. Les chats avaient, dans cette société, une telle valeur, qu'on leur consacrait des cimetières entiers et plus: on pouvait même en faire des momies, ce qui nous permettait de les conserver et de ne pas les oublier.

Mme Smith, l'institutrice, fera une lecture très juste du désarroi de Verity, lecture fondée dans un premier temps sur un malentendu. Nous sommes en pleine leçon sur l'Égypte ancienne. Mme Smith a bien noté le changement d'attitude chez Verity qui, aujourd'hui, dort littéralement sur sa table. Après la classe, elle lui demande ce qui se passe. Verity lui parle de ses cauchemars et cet échange s'ensuit:

Oh dear! s'exclame Mme Smith, did you tell your mum? My mum's dead. (32)

Ignorant que la mort de la mère remonte à la naissance de Verity, Mme Smith explique ainsi le chagrin et le malaise de son élève. Ce qui est en somme assez près de la vérité. « Did you tell your mum » vient combler le fossé séparant Verity du moment de sa naissance, la séparant de sa mère et séparant la vie de la mort. « Did you tell your mum » évoque l'urgence de la parole dans la situation d'inquiétude qui est celle de la fillette à ce moment-là, réveillant en elle une double impossibilité: celle du " dire » et celle de dire à sa mère.

Or, ce soir-là, en fouillant dans son armoire pour y prendre une couverture, Verity met la main sur quelque chose d'étrange, une sorte de fourrure qu'elle extirpe pour découvrir qu'il s'agit du corps mort de sa Mabel adorée. Son affliction n'a ici d'égal que l'ingéniosité qu'elle déploiera afin d'affronter la réalité d'une mort qu'elle a cette fois-ci bien en face. Elle décide de momifier Mabel, pour lui éviter d'abord d'être enterrée (comme sa mère), et ensuite pour lui permettre de demeurer dans un état permanent de conservation.

Verity met son plan à exécution, dans le plus grand secret, évitant à quiconque de se confronter tout comme elle à la réalité. Elle use des sels de bain à la lavande comme agent de conservation, découpe des vieux tissus pour momifier la bête, et demeure anxieuse tout au long de l'opération. Puis, elle place la momie Mabel dans son sac de sport qu'elle dépose dans son armoire. Ce chat momifié, ce cat mummy joue ainsi le rôle d'articulation langagière entre le chat et la mère. Momifier le chat reviendra donc à préserver la mère, à l'encrypter, tel dans le phénomène d'incorporation que l'on retrouve dans le deuil. À cet égard, l'étude de l'Égypte ancienne, avec ses pyramides, ses momies et ses sarcophages, est une réelle aubaine fantasmatique pour Verity.

La disparition de Mabel réveille en chacun le triste souvenir de la disparue, et cet événement fait basculer le cours jusque-là normal, ou à tout le moins maîtrisé, des choses. Verity cherchait une manière « to preserving Mabel for ever » (40). Cette manière est la momification: « I would make Mabel into 
a mummy! » Elle transformera son chat, donc, en maman: « Then I could still hold her in my arms and tell her I loved her and whisper messages to my mum » (40). Le discours devient ici explicite: un chat mort devient une mère objet, et l'on préservera en somme le chat sous son emballage de mère. La mort du chat est une résurgence de la mort de la mère, mais en fait, d'une mort tue.

C'est précisément pourquoi Verity ne dit à personne qu'elle a trouvé le chat mort. Elle a chèrement appris qu'on ne parle pas des morts, parce que cela bouleverse tout le monde. Mais pour elle, en fait, le bouleversement est venu du fait de n'avoir jamais pu en parler.

Or, parler de la mort est une manière de se poser en tant que vivant, une façon de baliser la vie elle-même:

... Nous parlons toujours de la mort sans y croire, bien que nous sachions tous qu'elle donne sens à notre vie, et que si elle n'était pas au bout, nous ne saurions même pas que nous sommes vivants! (Dolto 1998, 11).

Là se tient toute la vérité de l'enfant. La mort tue n'en est pas moins présente. Elle ne peut avoir pour Verity que l'allure d'une momie, d'une « mummy », d'une maman.

Venue chez Verity pour s'enquérir de la santé de l'enfant, l'institutrice assiste à la découverte, par la grand-mère horrifiée, du chat momifié et en processus de liquéfaction. Témoin de cette situation tragi-comique, c'est elle qui soufflera à l'oreille du père l'importance d'évoquer la mort de la mère et de susciter à son propos des échanges plus fréquents entre la fillette et sa famille. Enlever, en quelque sorte, les bandelettes ayant servi à occulter la mort de la mère.

Si nous avons souffert d'un deuil, écrit encore F. Dolto, cela fait partie de l'histoire de la famille et de ceux qui naîtront après, ça leur appartient car ce mort qui a vécu fait partie de la richesse vivante, symbolique de la famille... $(1998,29)$.

La sépulture du chat devient donc la possibilité du deuil et redonne enfin la parole à chacun concernant la mère morte. Mabel est au centre du drame tu: parler au chat pour rompre le silence autour de la mort; momifier le chat pour prolonger l'idée d'un événement enfoui, caché dans le passé familial. L'enfant, par son geste, fait en sorte que se réinscrive la mère dans le paysage familial. Elle en permet, à travers Mabel, la ré-appropriation symbolique.

Les morts sont parmi nous; ils ne peuvent être rayés d'entre les vivants. Ce silence entretient le mensonge de la vie éternelle, l'illusion de l'immortalité. Mais aussi, il empêche la résolution du deuil et alourdi le passé de dépouilles innommables. L'histoire de The Cat Mummy montre combien la petite fille ne peut être dupe de la mascarade du silence. 